when $\mathrm{H} 2 \mathrm{~B}$ showed a H2A-like aggregated pattern in the nuclei. The cytoplasmic accumulation of $\mathrm{H} 2 \mathrm{~B}$ was confirmed also by mass spectrometric identification of elevated levels of H2B following Dox treatment in the dechromatinized samples. Conclusions: Anthracyclines are widely used anti-cancer drugs exhibiting pleiotropic effects. At the chromatin level these include topoisomerase inhibition, DNA intercalation, aggregation of chromatin, histone eviction as well as direct binding to histones. The above large-scale effects were detected already at Dox concentrations that overlap serum peak levels reached in the typical clinical setting and therefore can be a factor both in the anticarcinogenic mechanism and in the side-effects of this anthracyclin.

This work has been supported by OTKA K128770 and GINOP-2.3.2-15-2016-00044.

doi: http://dx.doi.org/10.7124/bc.0009F2

\section{O-1. The role of the nuclear lamina in cell migration: the connection with aging and metastasis}

N. L. Ovsiannikova ${ }^{1,2 *}$, S. V. Lavrushkina ${ }^{3}$, A. S. Yudina ${ }^{1}$, O. S. Strelkova ${ }^{2}$, O. A. Zhironkina $^{2}$, I. I. Kireev ${ }^{2,3,4}$

${ }^{1}$ Faculty of Bioengineering and Bioinformatics, Lomonosov Moscow State University, Moscow, Russia; ${ }^{2}$ A.N. Belozersky Institute of Physical and Chemical Biology, Lomonosov Moscow State University, Moscow, Russia, ${ }^{3}$ Faculty of Biology, Lomonosov Moscow State University, Moscow, Russia; ${ }^{4}$ National Medical Research Center for Obstetrics, Gynecology and Perinatology named after
Academician V.I. Kulakov of Ministry of Healthcare of Russian Federation, Moscow, Russia nat.ovs94@gmail.com

Regulation of cancer cell migration remains one of the main tasks of practical biochemistry and molecular biology. One way to solve this problem is to control the elastic properties of nucleus since it is one as the biggest and stiffest compartment that determines the cell flexibility. At the same time, the nuclear rigidity is affected by the mechanical properties of the nuclear lamina (NL), a protein meshwork underlying the inner nuclear membrane. Ratio changing of its main structural components an A- and B-type lamins - is likely to be a key factor in the mechanical properties of nucleus. Furthermore, progerin, a mutant form lamin A, which induces Hutchinson Gilford Progeria Syndrome, is involved in aging by accumulation in NL, leading to nuclear stiffness increasing. However, how progerin presence in NL and changing of lamins ratio effect on cell migration processes is still unclear. To estimate the effect of lamins ratio changing on cell migration, we obtained HT1080 cell lines expressing GFP-lamin A, GFP-progerin and shRNA LMNA, which specifically prevents the production of lamin A. Cell migration ability was estimated by using scratch and transwell (with 3 and $8 \mu \mathrm{m}$ pore size) assays. The changing of NL composition is turned out to have no effect on migration in unrestricted space (scratch assay). Both overexpression of lamin A and progerin expression reduce the efficiency of cell migration through $3 \mu \mathrm{m}$ pore size membranes. Meanwhile, the suppression of lamin A expression has no effect on migration in a transwell. Our data, especially, from scratch assay show the absence of toxic effects of 
transfection on cell activity and migration in unrestricted space and through pores are larger than nucleus. The presence of various lamin A isoforms in NL is likely to lead to a reduction of migration ability due to the increase of the nuclear rigidity. The absence of effect of lamin A knockdown on migration may be explained that HT1080 cell line is supposed to have initially high migration ability and, possibly, more elastic NL and, consequently, it has originally reduced lamin A level. Therefore our next target is to estimate the level of lamin A isoforms by RT-PCR and WB and to conduct similar experiments by using other cell lines.

This work was supported by Russian Science Fund (RSF) № 17-15-01290.

doi: http://dx.doi.org/10.7124/bc.0009F3

\section{P-1. Electron microscopic approaches in studies of lipidic structures in the cell nucleus}

\author{
Vlada Philimonenko ${ }^{1,2}$, Zuzana Dolejšíi ${ }^{2}$, \\ Margarita Sobol ${ }^{1}$, Dominik Pinkas ${ }^{2}$, Toyo- \\ shi Fujimoto ${ }^{3}$, Pavel Hozák ${ }^{1}$ \\ ${ }^{1}$ Department of Biology of the Cell Nucleus, Insti- \\ tute of Molecular Genetics ASCR, Prague, Czech \\ Republic; ${ }^{2}$ Microscopy Centre, Electron Micros- \\ copy Core Facility, Institute of Molecular Genetics \\ ASCR, Prague, Czech Republic; ${ }^{3}$ Department of \\ Molecular Cell Biology, Nagoya University Gradu- \\ ate School of Medicine, Nagoya, Japan \\ vlada@img.cas.cz
}

So far, mostly protein complexes have been found as important for this ordering. We have described novel structures containing phosphatidylinositol 4,5-bisphosphate (PIP2) which seem to contribute as well. Ultrastructural studies demonstrate the PIP2-positive structures propagating through the nucleolus into the nucleoplasm where PIP2 is enriched in interchromatin granules, and also form previously undescribed 70-100 nm roundish "nuclear lipid islets" (NLI). Electron microscopy (EM) is essential for thorough characterization of the structure and molecular composition of this novel nuclear compartment. The usage of appropriate method for EM sample preparation is a crucial step as lipidic structures are potentially predisposed to extraction or translocations. Aims: To verify the results of ultrastructural localization of PIP2 obtained by several approaches including chemical fixation and cryoimmobilization followed by different immunolabeling techniques. Methods: We employed quick-freezing - freeze fracture replica labelling (QF-FRL), where the biological material is fixed physically - by high-pressure freezing, and subsequently mechanically - by coating with carbon/platinum layer. Due to it, QFFRL fixes also lipids and the samples are considered to be closer to the native state as compared to other techniques. Results: We were able to immunolabel PIP2 present in membranes as well as PIP2 which is localized in non-membranous compartments. NLI in the nucleoplasm were of the same size and shape as revealed by other techniques. Conclusions: The results demonstrate that NLI are stable structures resistant to aldehyde fixation and extraction, which is possibly ensured by interactions of lipidic components with proteins.

GACR (15-08738S, 19-05608S), TACR (TE01020118), CAS (JSPS-18-18), institutional grant (RVO: 68378050), MEYS CR (LM2015062), OPVVV (CZ.02.1.01/0.0/16 013/0001775). 